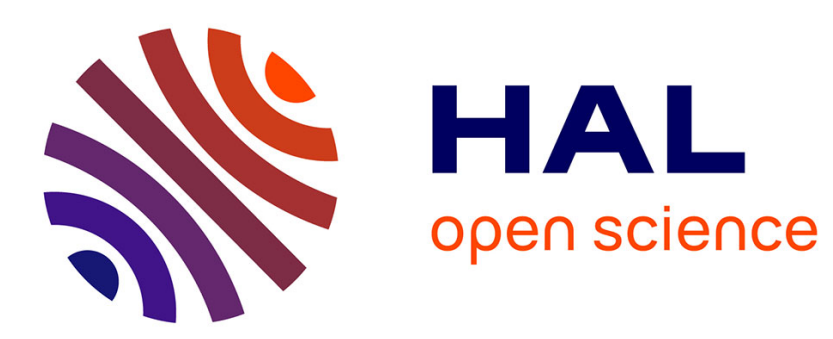

\title{
Optimal Transmission Radius for Energy Efficient Broadcasting Protocols in Ad Hoc and Sensor Networks
}

François Ingelrest, David Simplot-Ryl, Ivan Stojmenovic

\section{To cite this version:}

François Ingelrest, David Simplot-Ryl, Ivan Stojmenovic. Optimal Transmission Radius for Energy Efficient Broadcasting Protocols in Ad Hoc and Sensor Networks. IEEE Transactions on Parallel and Distributed Systems, 2006, 17 (6), pp.536-547. 10.1109/TPDS.2006.74 . inria-00112047

\section{HAL Id: inria-00112047 https://hal.inria.fr/inria-00112047}

Submitted on 7 Nov 2006

HAL is a multi-disciplinary open access archive for the deposit and dissemination of scientific research documents, whether they are published or not. The documents may come from teaching and research institutions in France or abroad, or from public or private research centers.
L'archive ouverte pluridisciplinaire HAL, est destinée au dépôt et à la diffusion de documents scientifiques de niveau recherche, publiés ou non, émanant des établissements d'enseignement et de recherche français ou étrangers, des laboratoires publics ou privés. 


\title{
Optimal Transmission Radius for Energy Efficient Broadcasting Protocols in Ad Hoc and Sensor Networks
}

\author{
François Ingelrest, David Simplot-Ryl and Ivan Stojmenović
}

\begin{abstract}
We investigate the problem of minimum energy broadcasting in ad hoc networks where nodes have capability to adjust their transmission range. The minimal transmission energy needed for correct reception by neighbor at distance $r$ is proportional to $r^{\alpha}+c_{\mathrm{e}}$, $\alpha$ and $c_{\mathrm{e}}$ being two environment-dependent constants. We demonstrate the existence of an optimal transmission radius, computed with a hexagonal tiling of the network area, that minimizes the total power consumption for a broadcasting task. This theoretically computed value is experimentally confirmed. The existing localized protocols are inferior to existing centralized protocols for dense networks. We present two localized broadcasting protocols, based on derived 'target' radius, that remain competitive for all network densities. The first one, TR-LBOP, computes the minimal radius needed for connectivity and increases it up to the target one after having applied a neighbor elimination scheme on a reduced subset of direct neighbors. In the second one, TRDS, each node first considers only neighbors whose distance is no greater than the target radius (which depends on the power consumption model used), and neighbors in a localized connected topological structure such as RNG or LMST. Then, a connected dominating set is constructed using this subgraph. Nodes not selected for the set may be sent to sleep mode. Nodes in selected dominating set apply TR-LBOP. This protocol is the first one to consider both activity scheduling and minimum energy consumption as one combined problem. Finally, some experimental results for both protocols are given, as well as comparisons with other existing protocols. Our analysis and protocols remain valid if energy needed for packet receptions is charged.
\end{abstract}

Index Terms-Ad Hoc Networks, Sensor Networks, Energy Efficient Broadcasting, Optimal Transmission Radius, Localized Communication Protocols.

\section{INTRODUCTION}

Ad hoc networks are autonomous and decentralized networks, composed of possibly mobile devices such as

F. Ingelrest and D. Simplot-Ryl are with IRCICA/LIFL, INRIA futurs, University of Lille 1, France. E-Mail: \{Francois.Ingelrest, David.Simplot\}@lifl.fr

Ivan Stojmenović is with Computer Science, SITE, University of Ottawa, Ontario K1N 6N5, Canada. E-Mail: ivan@site.uottawa.ca sensors, laptops or PDAs. Communications occur over a radio channel, ranges are thus restricted and only close nodes can communicate to each other. As the considered devices rely on batteries with limited capacity, the most important criterion when designing communication protocols is obviously energy conservation.

In this paper, we are interested in the broadcasting task, also referred to as flooding, that consists of sending a unique message from an arbitrary host to all the other ones in the network. This process can be used for many purposes, like publication of services or route discovery, as defined in DSR (Dynamic Source Routing) [1]. In ad hoc networks, as communication ranges are limited, many nodes must retransmit the packet to obtain a total coverage of the network. The simplest way to perform broadcasting is that each host relays once the message. If there exists a path between the source mobile and any other one, a total coverage is ensured. This method, known as Blind Flooding, is easy to implement but unfortunately leads to a huge and useless energy consumption:

- A lot of 'redundant' packets are generated, because nodes relay blindly even if their neighborhood has already been covered by other transmissions.

- If nodes can control their transmission range (and therefore their spent energy), it is useless in most cases to emit with a full power.

We consider the minimum energy broadcasting problem, where nodes adjust their transmisison power so that each node receives the packet and the total energy consumed by all nodes is minimized. The problem is known to be NP-complete [2] and most of the proposed solutions are centralized [3], that is a global knowledge of the network is needed to apply them. This is inefficient in a decentralized network, as many messages are to be exchanged between nodes to obtain such knowledge. Mobility or changes in activity status cause frequent changes in network topology. Centralized protocols are thus unusable in a practical context. Therefore, it is preferable to consider localized protocols, in which 
nodes make decisions based solely on the knowledge of their 1-hop (direct neighbors).

Since energy consumption depends on transmission ranges, a straightforward way to minimize it is to use radii as small as possible at each node. This observation was explored and applied in many previously proposed protocols. However, a commonly accepted energy model adds a constant to the consumption, regardless of the chosen radius, to take into account miscellaneous costs (such as minimal power needed for correct signal processing). Considering this, minimizing the energy consumption at each node may not be an optimal behavior, because small radii require more nodes to participate in the broadcasting process to cover a given area. This may lead to an increased global energy consumption, although each node has tried to minimize its own consumption. The problem is especially notable in dense networks, with small distances to the nearest neighbors. The previously proposed localized minimum energy broadcasting protocols [4] were based on covering the closest neighbors in a connected topology, and were therefore inferior to the centralized protocols for dense networks.

We present here the concept of an optimal transmission radius, whose length balances the energy consumption at each node and the number of needed relays. We first show how this radius is theoretically computed by using a hexagonal tiling of the network area. Then we present two localized broadcasting protocols that make use of this concept of optimal radius:

- The first one, named TR-LBOP (Target Radius LMST Broadcast Oriented Protocol), is a neighbor elimination scheme applied to a reduced subset of direct neighbors. Needed radii are modified to fit the desired value whenever it is possible.

- The second one, named TRDS (Target Radius and Dominating Sets based protocol), adapts the network topology and computes a connected dominating set, so that the distance between direct dominant neighbors is as near as possible to the optimal radius.

The rest of this paper is organized as follows. In the next section, we give the needed preliminaries on network and energy models. Section III proposes a literature review of existing energy efficient protocols for ad hoc networks. In Section IV, the concept of an optimal transmission radius and its computation are considered, while in Section V we present the two localized broadcasting protocols that make use of a target radius parameter. Then, Section VI gives some experimental results and compares our two protocols to other existing ones in the literature. We finally conclude in Section VII.

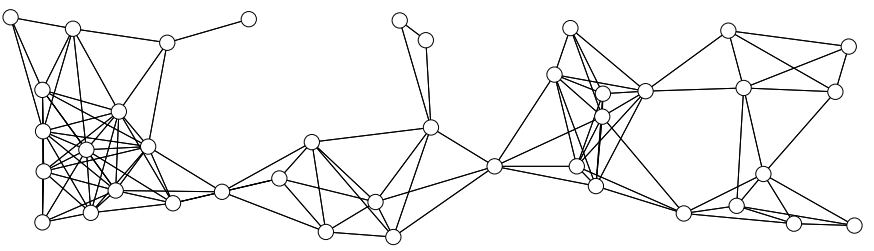

Fig. 1. Example of a unit graph.

Preliminary versions of portions of this article appeared in [5], [6].

\section{Preliminaries}

We represent an ad hoc network by a graph $G=$ $(V, E)$ where $V$ is the set of nodes (the mobiles) and $E \subseteq V^{2}$ is the set of edges that gives the available communications: $(u, v)$ belongs to $E$ means that $v$ is a physical neighbor of $u$, i.e. $u$ can directly send a message to $v$. Let us assume that the maximum range of communication, denoted by $R$, is the same for all vertices and that $d(u, v)$ is the Euclidean distance between $u$ and $v$. The set $E$ is then defined as follows:

$$
E=\left\{(u, v) \in V^{2} \mid d(u, v) \leq R\right\} .
$$

So defined graph is called the unit graph, with $R$ as its transmission radius. An example of such a graph is given in Figure 1. Each node $u \in V$ is assigned a unique value to be used as an identifier (id), so that the identifier of $u$ is denoted by $\operatorname{id}(u)$. We also define the neighborhood set $N(u)$ of a vertex $u$ as:

$$
N(u)=\{v \mid(u, v) \in E\} .
$$

The size of this set, $|N(u)|$, is also known as the degree of $u$. The density of the graph is the average degree for each node. Note that $(u, u)$ is not in $E$.

Nodes in a minimum energy broadcasting protocol need position information about their neighbors. The common method used to gain this knowledge is the use of special short messages named HELLO messages that are periodically emitted by each node, announcing their own position. To find their position, nodes may use a location system like the GPS. Other miscellaneous positioning or distance measurement systems can be found in the literature [7]-[10].

We assume that all packets are of the same size (number of bits). In the most commonly used energy model, the measurement of the energy consumption of network interfaces when transmitting a fixed size message depends on the range $r$ of the emitter $u$ : 


$$
E(u)= \begin{cases}r(u)^{\alpha}+c_{\mathrm{e}} & \text { if } r(u) \neq 0, \\ 0 & \text { otherwise, }\end{cases}
$$

$r(u)$ being the transmitting range of $u$ and $c_{\mathrm{e}}$ a constant that represents an overhead due signal processing. The model $\alpha=4, c_{\mathrm{e}}=10^{8}$ is derived from a work by Rodoplu and Meng [11], and it seems realistic enough to be used as a reference. These values are expressed in arbitrary units, and can be converted into any given units by using the corresponding multiplication factor.

Nodes also consume some energy upon the reception of a message. This consumption $c_{\mathrm{r}}$ is constant, regardless of the distance between the emitter and the receiver. The reference value generally used is one third of the energy consumed by a 100-meter emission, that is $c_{\mathrm{r}}=\frac{1}{3} \times$ $\left(100^{\alpha}+c_{\mathrm{e}}\right)$. In the previously stated model, this gives $c_{\mathrm{r}}=\frac{2}{3} \times 10^{8}$.

\section{RELATED WORK}

A straighforward protocol to broadcast a message is called Blind Flooding: each node simply relays once the message to its neighborhood. Many solutions have been proposed to replace this inefficient method, and mainly two families can be distinguished:

- The first one reduces the number of needed emissions to obtain a total coverage.

- The second one considers radius adjustment with suitable hardware to further reduce energy consumption. The previous method can be seen as the discrete version of this one: nodes can only choose between 0 and the maximum value for their radius, while here it can be any value in this interval.

All these solutions consider omnidirectional antennas, that is a single transmission is received by all the neighboring nodes located within selected transmission range. We adopt this model in this article. There also exists some solutions that consider directional antennas [12], for which an angle of emission can be chosen.

\section{A. Minimizing the Quantity of Transmissions}

A remarkable protocol that belongs to this family is named MPR (Multipoint Relay Protocol) and has been proposed by Qayyum et al. [13]. It is a greedy heuristics which makes nodes select their own relays before retransmitting. This selection, which is forwarded with the broadcasting packet, is composed of an optimal subset of direct neighbors that entirely covers the 2-hop neighborhood. Nodes that receive this packet but do not have been selected do not relay it. This protocol is used in an IETF standardized routing protocol named OLSR (Optimized Link State Routing) [14].

The NES (Neighbor Elimination Scheme) was independently proposed in [15] and [16]. In this scheme, nodes do not relay the message immediately but monitor their physical neighborhood for a given time. Each neighbor that receives a copy of the same message is eliminated from an internal uncovered neighbors list. If this list becomes empty before the timeout occurs, the retransmission is canceled. This method has been further improved by other protocols like RRS ( $R N G$ Relay Subset) [17], which reduces the set of monitored neighbors (and thus the probability of retransmitting) by using a special (simplified) graph named RNG (Relative Neighborhood Graph) [18].

Finally, some broadcasting protocols are based on the computation of a subset of $V$, denoted by $V_{\text {dom }}$, which satisfies two properties:

- All nodes in $V$ either belong to $V_{\text {dom }}$ or are directly connected to a node in $V_{\text {dom }}$ (i.e. $V_{\text {dom }} \cup$ $\left.N\left(V_{\text {dom }}\right)=V\right)$. Such a subset is a dominating one.

- It is connected (i.e. there exists a path between any two nodes in $\left.V_{\text {dom }}\right)$.

Finding the smallest possible connected dominating set is a NP-complete problem even if a global knowledge of the network is provided [19]. Despite such difficulty, some localized algorithms to compute an efficient suboptimal set have been proposed and can thus be used in ad hoc networks. Amongst them is the Generalized Self Pruning Rule proposed by Dai et al. [20]. This algorithm computes an efficient connected dominating set by using solely the knowledge of the physical neighborhood. However, it is based on a 'marking scheme' where nodes exchange several messages to inform about their status at various stages of the protocol. The protocol was modified in [21] to avoid message exchanges. Therefore each node can decide whether or not it is in dominating set without exchanging any messages with neighbors, following the protocol in [21]. This rule was computationally simplified in [22], and we describe here only that variant. Note that [20], [21] and [22] generate the same dominating sets, but with different communication and computation complexities. First, each node checks if it is intermediate, that is, whether it has at least two neighbors not directly connected. Then each intermediate node $A$ constructs a subgraph $G_{\mathrm{h}}$ of its neighbors with higher priorities: in the graph composed by $A$ and its neighborhood, each node which has a lower priority than $A$ is removed, $A$ is also removed, as well as the corresponding edges. The resulting subgraph is denoted by $G_{\mathrm{h}}$. If the latter 
is empty or disconnected then $A$ is in the dominating set. If $G_{\mathrm{h}}$ is connected but there exists a neighbor of $A$ which is not neighbor of any node from $G_{\mathrm{h}}$ then $A$ is in the dominating set. Otherwise $A$ is covered and is not in the dominating set. Dijkstra's shortest path algorithm can be used to test the connectivity (it is performed locally at each node). Non-intermediate nodes are never dominant. To broadcast a message, dominant nodes are the only needed relays to cover the whole network.

The priority of a node can be represented by any collection of values: comparisons are then made on the primary key, if they are equal on the secondary key and so on. In the original version of this algorithm, the id of nodes was used as a simple key, but many other metrics can be found in [23].

\section{B. Adjusting Radii}

Several broadcasting protocols based on adjusting transmission radii are based on the computation of a connected subgraph of the original network graph $G$. Edges of the considered subgraph are used to determine communication radii. The following three subgraphs are used:

- The MST (Minimum Spanning Tree) is used in [3]. It is a tree connecting all nodes whose total edge weight is minimized. The weight is normally edge length, and this tree has short edges.

- The RNG (Relative Neighborhood Graph), which removes the longest edge of any triangle in the graph and has an average degree of 2.6. This graph was introduced by Toussaint [18].

- The LMST (Local Minimum Spanning Tree), in which nodes compute the MST of their physical neighbors and keep only edges that are selected by both endpoints in their respective MSTs. This graph was proposed recently by $\mathrm{Li}$ et al. [24]. It has an average degree of 2.04 and has been proven to be a subgraph of the RNG [4]. It is proven in [25] that MST is a subset of LMST.

Figure 2 illustrates these subgraphs. It can be noticed that only the MST requires centralized protocol: the knowledge of the whole topology of the network is required for its computation. The RNG can be locally computed without requiring any message exchange. The computation of LMST requires one message from each node in order to remove asymmetric links.

Wieselthier et al. defined in [3] a topology control algorithm which is based on the MST. Each node chooses a radius that covers only neighbors in the MST. Since, by its construction, this graph is connected, the new graph derived from this range assignment is also always connected. This method offers good performance (except for very dense networks), but is costly for implementation in ad hoc networks because of centralized computation of MST. Other protocols that use locally defined graphs instead of the MST have since been proposed: the protocol RBOP (RNG Broadcast Oriented Protocol) [26] uses the RNG while LBOP (LMST Broadcast Oriented Protocol) [4] uses the LMST.

In the same paper, Wieselthier et al. also defined a centralized protocol named BIP (Broadcast Incremental Power). This heuristics constructs from a source node a broadcasting tree that spans the network. Nodes are added one by one to the tree by choosing the less expensive action: either a node that is already emitting increases its radius or a node that is not emitting starts a new transmission. The 'price' of an action is the additional power needed to cover one more node. A node cancels its transmission if it notices that the circle covered by its transmission is completely inside another circle covered already by a transmitting neighbor.

\section{The Concept of AN Optimal TRANSMission RADIUS}

Our algorithms are based on the observation that it is not always suitable to minimize the transmission radius. Indeed, the number of transmissions needed to cover the network is inversely proportional to the (square of the) range used. Therefore, for large $c$ in $r^{\alpha}+c$, many transmissions over short edges are energy consuming due to multiples of $c$. Large radius also causes large power consumption. In this section, we show that there exists an optimal radius, depending on the energy model.

Let us consider a rectangular area $S$ on which some emitting nodes are to be placed. These nodes will have to perform a flooding, so that the whole area will be covered. Emissions are done with a range that is going to be determined, the goal being to perform the task while minimizing the energy consumption. There are thus two related parameters to set:

- The number of nodes, denoted by $n$.

- The range used for emissions, denoted by $r$.

The main problem is the placement of the emitting nodes. We choose a hexagonal tiling, that is the area is divided into several hexagons, nodes being placed on vertices. Obviously, the distance between emitters should be exactly $r$ to avoid having holes and 'useless' nodes (i.e. whose transmission area is entirely covered by other nodes), so we choose $r$ as the length of the side of an hexagon. The quantity of vertices $n$ (i.e. nodes) now depends on the value of $r$, as illustrated by Figure 3 . 


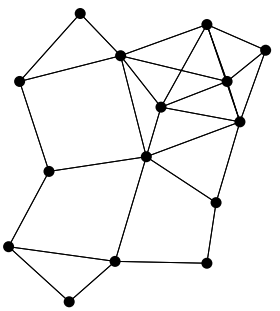

(a) Unit graph

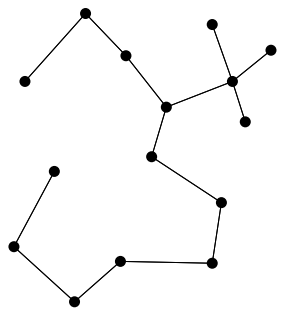

(b) MST

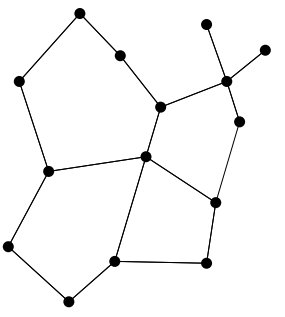

(c) LMST

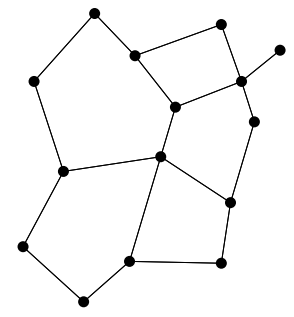

(d) RNG

Fig. 2. Example of an unit graph and its associated subgraphs.
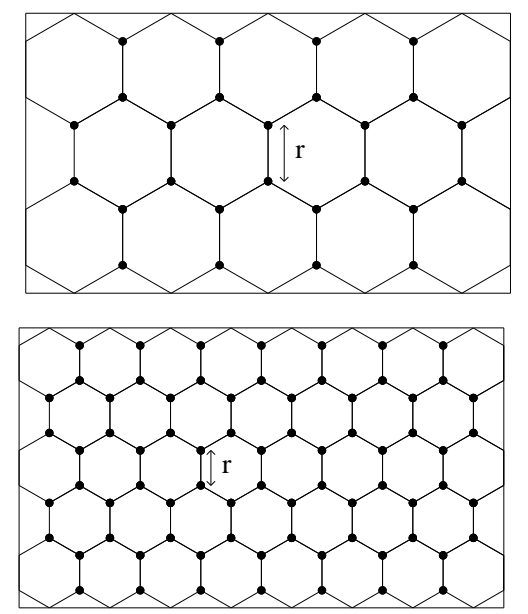

Fig. 3. Hexagonal tiling of the area $S$ for two different values of $r$.

The edge length (and also transmission radius) $r$ is a variable. The goal is to find its value that minimizes overall energy consumption, and consider that value as the network parameter. As suggested previously, two behaviors can be distinguished here:

- Using a high value for $r$ would make nodes cover a large part of the area, thus decreasing the value of $n$.

- On the contrary, using a low value for $r$ would increase the value of $n$.

So we just have to find the optimal distance between nodes, that is the value of $r$ that leads to the minimal energy consumption for the considered flooding. Knowing that $r$ is the exact distance between two neighboring vertices, we can easily compute the needed quantity of nodes to cover the whole area. To do this, we only have to compute how many hexagons, denoted by $h$, fit on our area of surface $S$ :

$$
\mathrm{h} \simeq \frac{\text { Area of the region }}{\text { Area of a hexagon }}=\frac{S}{\frac{3}{2} r^{2} \sqrt{3}}=\frac{2 S}{3 r^{2} \sqrt{3}} .
$$

To tile the area, two nodes have to be placed per hexagon (since each hexagon has six nodes, and each node is common to three hexagons), the number of nodes $n$ is then:

$$
n=2 h=\frac{k}{r^{2}}, \quad k=\frac{4 S}{3 \sqrt{3}},
$$

$k$ being a constant value that does not depend on the value of $r$.

\section{A. An Ideal Computation of the Optimal Transmission Radius}

In this part, we do not consider the loss of energy of nodes due to the reception of a message. Thus, by using (1), we can define the consumption of a blind flooding with a transmission radius $r$ to be:

$$
\mathrm{PC}(r)=n\left(r^{\alpha}+c_{\mathrm{e}}\right)=\frac{k\left(r^{\alpha}+c_{\mathrm{e}}\right)}{r^{2}} .
$$

The optimal transmission radius $r$ is simply the one that gives the minima of the function $\mathrm{PC}(r)$, which are determined by $\alpha$ and $c_{\mathrm{e}}$. Given that $\alpha \geq 2, c_{\mathrm{e}} \geq 0$ and $r>0$, there are only a few cases to enumerate:

- $\alpha=2, c_{\mathrm{e}}=0 \quad \mathrm{PC}(r)=k$, regardless of $r$.

- $\overline{\alpha=2, c_{\mathrm{e}} \neq 0} \quad \mathrm{PC}(r)=k\left(1+c_{\mathrm{e}} \cdot r^{-2}\right)$ which has no minimum, but the greater the $r$, the smaller the consumption. That is, a single transmission reaching all network nodes appears energy optimal.

- $\alpha>2, c_{\mathrm{e}}=0 \quad \mathrm{PC}(r)=k r^{\alpha-2}$ which does not have a minimum when $r>0$, but the smaller the $r$, the smaller the consumption. That is, selecting nearest neighbors, with preserved connectivity, as explained, appears optimal in this case.

- $\alpha>2, c_{\mathrm{e}} \neq 0 \quad \mathrm{PC}(r)=k\left(r^{\alpha-2}+c_{\mathrm{e}} r^{-2}\right)$ which has a minimum. We have:

$$
\mathrm{PC}^{\prime}(r)=k\left((\alpha-2) r^{\alpha-3}-2 c_{\mathrm{e}} r^{-3}\right),
$$

which reaches zero when :

$$
r=\sqrt[\alpha]{\frac{2 c_{\mathrm{e}}}{\alpha-2}} .
$$




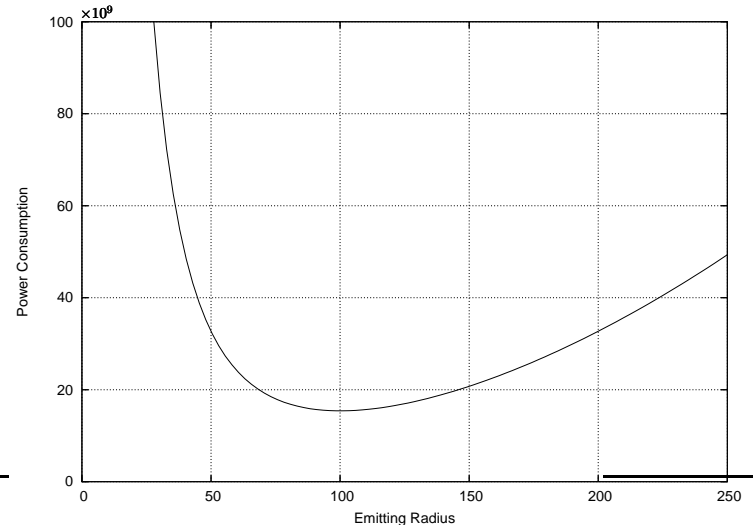

Fig. 4. Power consumption with $\alpha=4$ and $c_{\mathrm{e}}=10^{8}$.

Considering the fourth case with $\alpha=4$ and $c_{\mathrm{e}}=10^{8}$, Figure 4 gives the power consumption of a flooding over our rectangular area. It clearly shows that the optimal Below this value, there are too many emitting nodes, making the constant $c_{\mathrm{e}}$ a problem while a greater radius makes the constant $\alpha$ a problem. It can be observed, however, that the function has small slope around the optimal radius, and deviation of up to $20 \%$ from the optimal radius does not have significant impact on the optimality. This is an encouraging observation, since in reality we do not have nodes at ideal hexagonal tiling, but selecting existing nodes nearby gives satisfactory approximations.

It can also be noticed that $\alpha=2$ brings special cases. In the first one $\left(c_{\mathrm{e}} \neq 0\right)$, the maximal radius must be used to minimize the energy consumption, while in the second one $\left(c_{\mathrm{e}}=0\right)$ the chosen radius does not influence the power consumption.

In a practical context, nodes spend some energy when they receive messages, as stated in Section II. Thus, each emission will be received by a number of neighbors that depend on the area covered by this emission, which itself depends on the chosen radius $r$. If $d$ is the original density when using the maximal range $R$, then the density $d(r)$ when using a radius $r$ is: radius $r$ is 100 , which is indeed a solution of $\mathrm{PC}^{\prime}(r)$.

\section{B. Consideration of the Power Consumption upon Re- ception}

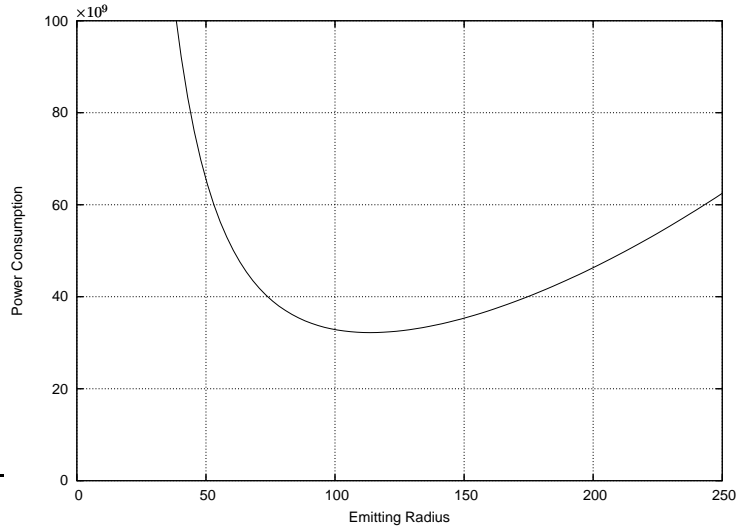

Fig. 5. Power consumption with $\alpha=4, c_{\mathrm{e}}=10^{8}$ and $c_{\mathrm{r}}=\frac{2}{3} \times 10^{8}$.

charge a reception to the emitter. The power consumption of the broadcasting then becomes:

$$
\mathrm{PC}(r)=\underbrace{n \times\left(r^{\alpha}+c_{\mathrm{e}}\right)}_{\text {Emissions }}+\underbrace{n \times c_{\mathrm{r}} \times(d(r)+1)}_{\text {Receptions }},
$$

which becomes:

$$
\mathrm{PC}(r)=k \times\left(r^{\alpha-2}+\left(c_{\mathrm{e}}+c_{\mathrm{r}}\right) r^{-2}+\frac{d c_{\mathrm{r}}}{R^{2}}\right) .
$$

Considering the case where $\alpha>2, c_{\mathrm{e}} \neq 0$ and $c_{\mathrm{r}} \neq 0$, the derivative of this function is:

$$
\mathrm{PC}^{\prime}(r)=k\left((\alpha-2) r^{\alpha-3}-2\left(c_{\mathrm{e}}+c_{\mathrm{r}}\right) r^{-3}\right)
$$

which reaches zero when

$$
r=\sqrt[\alpha]{\frac{2\left(c_{\mathrm{e}}+c_{\mathrm{r}}\right)}{\alpha-2}} .
$$

Figure 5 illustrates the power consumption of the blind flooding when considering the model of Rodoplu and Meng $\left(\alpha=4, c_{\mathrm{e}}=10^{8}, c_{\mathrm{r}}=\frac{2}{3} \times 10^{8}\right)$. Once again, there is a minimum value in the consumption, which happens this time with $r=113.62$.

If the transmitting node switches off its own receiver to avoid energy being used for the unnecessary reception, the ' +1 ' portion in the total power consumption formula $P C(r)$ will disappear, and the formula for the target radius becomes the same as in the case when reception power was not charged at all (that is, same as if we $d(r)=d \times \frac{\text { Area covered with radius } r}{\text { Area covered with radius } R}=d \times \frac{\pi r^{2}}{\pi R^{2}}=\frac{d r^{2}}{R^{2}}$ for this outcome. Consider a given non-transmitting node positioned anywhere in the hexagonal tiling. Regardless of the value of $r$, it receives fixed number of transmissions. Scaling hexagons around selected node scales equally the transmitting radius, therefore has no
Thus, a node emitting with a radius $r$ reaches $d(r)$ neighbors. We first consider the case when emitting node does not switch off its own receiver and therefore we 
impact on the number of receptions. More precisely, the whole area can be divided into some sectors, with given fixed number of receptions received in each sector, and that figure simply scales with $r$ without impacting the number of receptions. Consider now a transmitting node. If its own transmission is not charged, then the same conclusion about irrelevance of reception energy on the selection of the target radius is valid. However, if its own receiver is on then the total number of such receptions equals the total number of transmitting nodes, therefore $+c_{r}$ is added to $c_{e}$ in the formula for the target radius.

\section{TARget Radius Broadcasting Protocols}

Given all the previous results about the computation of an optimal radius, our goal is now to design efficient broadcasting protocols. The goal is to decide which nodes should relay the broadcasting message and with which radius.

We present here two protocols that make use of a target radius parameter in the designation of relays and radii:

- The first one is named TR-LBOP (Target Radius LMST Broadcast Oriented Protocol). Its concept is to compute the minimal needed radius for each node to preserve the connectivity, and to increase it up to the target one whenever it is possible.

- The second one is named TRDS (Target Radius and Dominating Sets based protocol). This protocol uses a topology control step to designate dominant nodes as relays with a distance between them as close as possible to the target radius.

Both of them use a parameter that we call a 'target radius', whose goal is to influence the distance between emitting nodes, thus controlling the topology. Depending on the considered energy model, the computed value of the optimal range should be used for this parameter, referred to as $T$.

\section{A. TR-LBOP}

We selected to modify the LBOP protocol [4] for three different reasons:

- It is localized.

- The Neighbor Elimination Scheme considers only nearest neighbors.

- It performs well when compared to centralized protocols like BIP, for graphs that are not very dense (where the impact of the target radius concept is negligible).

The principle of LBOP is as follows. Each node that receives the broadcasting message for the first time generates a list of its LMST-neighbors that have not received this message and starts monitoring the communications that occur in its neighborhood. Each time one of its LMST-neighbors receives the message, the corresponding node is removed from the list. After a given timeout, two cases can happen: if the list is empty, the retransmission is canceled, otherwise the message is relayed with the radius needed to cover the furthest neighbor in the list. This is a standard neighbor elimination scheme (NES) limited to LMST-neighbors.

As the node density increases, RNG and LMST neighbors are getting closer, and therefore LBOP reduces transmission radii. Rebroadcasting with a minimal radius is not always an optimal behavior, because too short radii require more nodes to act as relays. The constant energy charge for each transmission then leads to energy inefficient solution compared to BIP protocol, for example.

We apply the concept of optimal 'target' transmission radius to design a competitive minimum energy broadcasting protocol for all network densities. We modify some parts of the LBOP algorithm, so that each node increases its radius up to the target one when a retransmission is needed.

Each node $u$ has to manage two lists $L(u)$ and $L^{\prime}(u)$ during the NES. The first one, $L(u)$, stores the neighbors needed to keep the connectivity of the network. As with LBOP we use the LMST-subgraph, so that $L(u)$ contains neighbors $v$ of $u$ for which the edge $(u, v)$ belongs to $L M S T$. The list $L^{\prime}(u)$ stores every other neighbor of the node $u$ :

$$
\forall u \in V \quad L^{\prime}(u)=N(u) \backslash L(u) .
$$

During the NES, each neighbor that receives the broadcasting message is removed from the corresponding list ( $L$ if it is a LMST-neighbor, $L^{\prime}$ otherwise). Of course, the node $u$ can immediately remove the neighbor from which it received the message and their common neighbors which also received the same message, based on the transmission radius used.

When the timeout is up, two cases can happen:

- The list $L(u)$ is empty, in which case the retransmission is canceled, as with LBOP, because it is not needed to keep the connectivity.

- There is at least one node in $L(u)$, in which case the node $u$ has to rebroadcast the message to reach the nodes left in $L(u)$.

In the second case, when the retransmission is needed, we know that the node will have to support the cost of the constant $c_{\mathrm{e}}$. So, as explained previously, it can be more 'intelligent' to increase the needed radius up to the target 


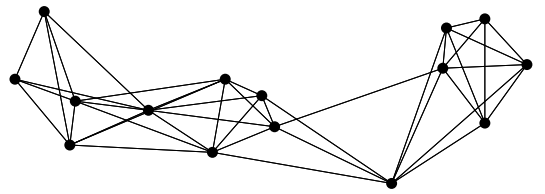

(a) $T=100$ meters
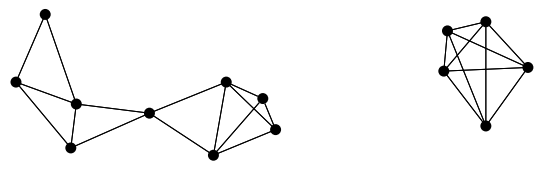

(b) $T=65$ meters

Fig. 6. Loss of connectivity with an adjusted radius.

one, when it is possible. We define two values, $D_{L}$ and $D_{L^{\prime}}$. The first one, $D_{L}$ is defined by:

$$
D_{L}=\max \{d(u, v) \mid v \in L(u)\},
$$

The second one, $D_{L^{\prime}}$ is defined by:

$$
\begin{aligned}
D_{L^{\prime}}=\{d(u, v) \mid v & \in L(u) \cup L^{\prime}(u) \wedge \\
\delta_{u v} & \left.=\min \left\{\delta_{u w} \mid w \in L(u) \cup L^{\prime}(u)\right\}\right\},
\end{aligned}
$$

where $\delta_{u v}=|d(u, v)-T|$.

In other words, the chosen distance is the length of the edge between the node $u$ and its non-reached neighbor which is the nearest one from $T$. The final chosen radius is simply:

$$
r(u)=\max \left\{D_{L}, D_{L^{\prime}}\right\} .
$$

In dense networks, this modification leads to a situation where nodes mostly emit with a radius as close as possible to $T$. The increased number of reached neighbors is balanced with the full neighbor elimination scheme of LBOP, so that the number of relays does not increase dramatically.

Experimental results for this protocol are given in Section VI.

\section{B. TRDS}

The main idea of this protocol is to reduce radii of nodes down to the target one. However, restraining radii in a localized manner is not that easy, as connectivity must be preserved. Figure 6 shows this problem with two different target radii. If nodes uniformly choose $100 \mathrm{~m}$. as their radius, the resulting network is connected, while it is no longer the case with $T=65 \mathrm{~m}$. This clearly illustrates the need for some nodes to use a longer radius to preserve the connectivity.

Our protocol uses a target radius and a locally defined connected graph, like the RNG or the LMST, to preserve the connectivity. Given these elements, the algorithm is divided into three steps:

1) Adapt the topology of the network so that each node chooses a radius as close as possible to $T$, while still maintaining the connectivity. This is achieved by constructing the subgraph where each node considers only neighbors in RNG (or LMST) and neighbors whose distance is no greater than the target radius.

2) Select dominant nodes to relay the message. A connected dominating set (CDS) is determined using constructed subgraph. Nodes not selected for CDS may be sent to sleep mode and periodically woken up for sending and receiving messages from associated dominating set nodes, if activity scheduling is also considered.

3) Perform the broadcasting over this new topology. Nodes in selected CDS remain active and apply TR-LBOP. If all nodes remain active then nodes not selected in dominating set do not retransmit, but impact the decisions of nodes from selected CDS.

\section{Topology Control using T}

Let $G=(V, E)$ be a connected graph. To preserve the network connectivity while modifying the transmission range of nodes, we compute a subgraph $G^{\prime}=\left(V, E^{\prime}\right)$, which has to be connected, sparse, bidirectional and computed locally. Some good examples of such graphs are the RNG or the LMST.

From $G, G^{\prime}$ and $T$, we compute for a node $u$ a range assignment $r(u)$ defined by:

$$
\begin{aligned}
\forall u \in V, \quad r(u)=\max & \{d(u, v) \mid v \in V \\
& \left.\wedge\left(d(u, v) \leq T \vee(u, v) \in E^{\prime}\right)\right\} .
\end{aligned}
$$

In other words, each node chooses a range that covers all its neighbors that belong to $G^{\prime}$ (to preserve connectivity) and also those that are closer than $T$ (these ones can belong to $G$ ). This graph is denoted by $G_{r}=$ $\left(V, E_{r}\right)$. The topology we keep is the symmetrical part of $G_{r}$; unidirectional links are simply removed. Each node should send a message containing the pre-selected transmission range in order to eliminate asymmetric links, and possibly adjust its transmission range afterwards. 


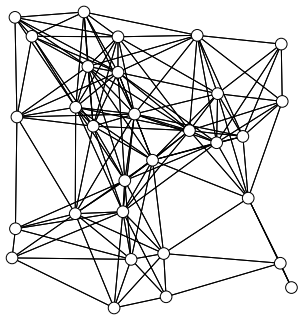

(a) Unit Graph

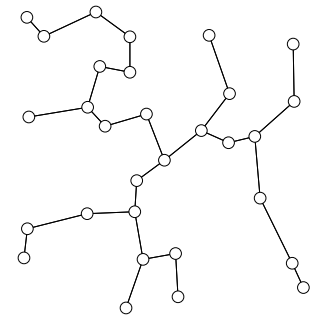

(b) LMST

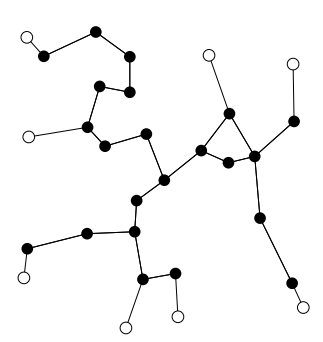

(c) $T=50$

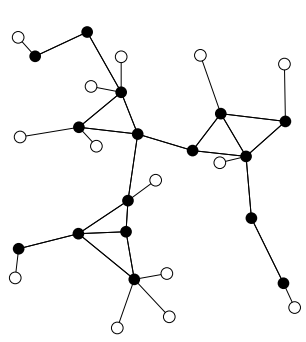

(d) $T=150$

Fig. 7. Unit graph with its $\operatorname{LMST}(R=250)$ and dominating sets after topology control with $T=50$ and $T=150$.

An asymmetrical graph could also be used if a suitable computation of a connected dominating set is applied. The removal of directional links may further reduce the radius needed for preserving the connectivity. We denote this graph by $G_{T}=\left(V, E_{T}\right)$ and it is defined by:

$E_{T}=E_{r} \cap E_{r}^{-1}, \quad E_{r}^{-1}=\left\{(u, v) \in V^{2} \mid(v, u) \in E_{r}\right\}$.

Edges that belong to the set $E_{r}$ are directed, $E_{r}^{-1}$ contains the same set of 'reversed' edges. By keeping the intersection of the two sets, we keep only the bidirectional links.

This new graph is obviously connected, since it contains $G^{\prime}$. After this step, each node has a radius as close as possible to $T$ with a connectivity preserved, of course as long as the original graph was itself connected.

\section{Energy Efficient Dominating Sets}

Given a connected graph $G_{T}$, obtained thanks to the first step, we now compute a connected dominating set to select which nodes should act as relays. To compute such a set, many algorithms have been proposed [20], [27], [28], and any of them can be used.

Hence, the set of vertices is now composed of two sets:

- The first one, $D$, is composed from dominant nodes. Only these nodes will have to relay messages.

- The second one, $\bar{D}=V \backslash D$, contains the rest of the nodes.

We associate each non-dominant node $u \in \bar{D}$ to its closest dominant neighbor. The latter, denoted by $p(u)$, is in charge of $u$, meaning it has to choose a radius that covers $u$ when this one has not already been covered. This coverage is needed if $u$ is awake, otherwise (if $u$ is sleeping), the distance to $u$ does not need to be considered in the broadcasting process. Note that, in our experiments, we assumed the need for coverage despite possible sleep status, since no significant differences were discovered.

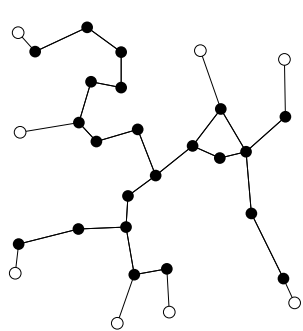

(a) $T=50$

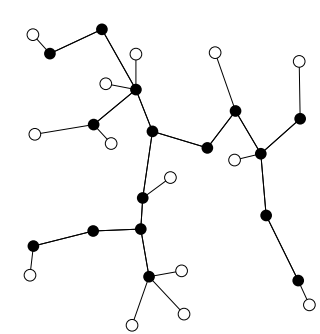

(b) $T=150$
Fig. 8. Final graphs with dominating sets and associated nondominant nodes.

For instance, Figure 7 shows an unit graph $(R=$ 250 ) with its associated (symmetrical) LMST. We have applied our topology control algorithm for two different values of $T$. For both cases, we have computed a dominating set by using the self-pruning generalized rule [20]. Black nodes are dominant, while white ones are non-dominant. Some edges have been removed for the sake of clarity: non-dominant nodes are only linked to their closest dominant node.

We further reduce the complexity of the dominant subgraph by computing the RNG of it. By using this graph, every dominant node has just to cover its dominant neighbors that belong to the RNG, which can furthermore reduce the needed radius in the process of broadcasting. For instance, Figure 8 gives the final graphs where we have computed RNG over dominant node subgraph of Figure 7. Note that one can also use LMST instead of RNG for the same purpose. However, this would require extra message from each node.

\section{The Broadcasting Process}

For a node $u$, the sets of dominant neighbors $N_{D}(u)$ and non dominant neighbors associated with it $N_{\bar{D}}(u)$ are defined by:

$\forall u \in V \quad\left\{\begin{array}{l}N_{D}(u)=\left\{v \mid v \in D \wedge(u, v) \in E_{T}\right\} \\ N_{\bar{D}}(u)=\left\{v \mid v \in \bar{D} \wedge u=p(v) \wedge(u, v) \in E_{T}\right\}\end{array}\right.$ 
Each node should send a message to its neighbors about its dominating set status in order to determine neighbors in the graph and properly select the target radius. The broadcasting algorithm proceeds as follows:

1) A dominant node $u$ that wishes to launch a broadcasting emits its message with the minimal range that covers $N_{D}(u)$ and $N_{\bar{D}}(u)$.

2) A non-dominant node that wishes to launch a broadcasting emits its message to its nearest (associated) dominant neighbor.

3) A dominant node $u$ that receives the message rebroadcasts it with the range which allows to cover non-covered nodes in $N_{D}(u)$ and $N_{\bar{D}}(u)$. It does not take into account neighbors that have been covered (according to the knowledge of the node, extracted from messages previously received) when it received the message.

4) A non-dominant node that receives the message never relays it.

\section{Vi. Performances Evaluation}

In this section, we give experimental results obtained with our own $\mathrm{C}++$ simulator for our proposed protocols TR-LBOP and TRDS, and comparisons with existing other protocols. For these comparisons, we chose:

- LBOP, as TR-LBOP is based on it.

- BIP, which is one of the best centralized solutions.

Our theoretical analysis shows that there is no mathematical or algorithmic difference between cases with and without reception charge, only a constant $c=c_{\mathrm{e}}+c_{\mathrm{r}}$ changes its value. Since constant is basically an arbitary number taken from some literature, the same diagrams for both cases can be used, and there is no need for separate experiments and separate explanations. We thus used the common parameters derived from the paper by Rodoplu and Meng [11], so that the values used are: $\alpha=4, c=10^{8}$.

As our goal is to compare the energy savings obtained by these protocols, we do not consider mobility here. So, in our simulations, the network is static and is always composed of 300 nodes randomly placed in a square area whose size is computed to obtain a given density. The MAC layer is assumed to be ideal, that is, no collision occurs when two nodes emit at the same time. The initial maximum communication radius $R$ is fixed to 250 meters. The timeout for the neighbor elimination scheme in TR-LBOP is randomly generated. For each measure, 250 broadcasts are launched and for each broadcast, a new connected network is generated. The average selected density in our experiments (for tables that do not use density as independent variable) is 50. Time complexities of algorithms like TRDS with LMST are quadratic in density: simulations done with density 100 are about 4 times slower than those with density 50 .

We define the efficiency of a protocol in terms of energy savings, so we could compute the power consumptions of each of them and compare them to each other. However, as each network is randomly generated for each iteration and protocol, the consumptions cannot be directly compared. Thus, we compute a ratio that we call EER (Expanded Energy Ratio), which represents the energy consumption of the considered protocol compared to the energy that would have been spent by a simple blind flooding for the same network. The value of EER is therefore defined by:

$$
E E R=\frac{E_{\text {protocol }}}{E_{\text {flooding }}} \times 100 .
$$

As our protocols are based on a target radius, we make comparisons by varying this parameter between 0 and the maximum range (which is set to $250 \mathrm{~m}$.). Each time, the protocol TRDS is used in conjunction either with the LMST or with the RNG for the topology control step.

Our main goal when designing the proposed protocols was to emit using the 'target' radius $T$. So, we first depict the effectiveness of this parameter on the topology control in our protocols in Figure 9, which gives in 9(a), for a range of different $T$ values, the average radius effectively used by nodes during the broadcasting. It works as intended, since we are easily able to control these radii, except for too low values, where nodes must use a longer one to keep the connectivity: $T$ is too low to keep the graph connected, and the distance between neighbors in the chosen subgraph (LMST or RNG in our experiments) is then used as a minimum value. We have therefore designed localized protocols where nodes apply preferred transmission radii.

In Figure 9(b), we focus on TRDS and give the average distance between dominant neighbors, depending on $T$. This distance is important, because in this protocols only dominant nodes (except possibly the source) will emit packets to perform the broadcasting, and the chosen radius will have to cover at least dominant neighbors. This again demonstrates that $T$ correctly influences the topology, since we are able to control the distance between dominant nodes. More precisely, it appears that the average distance between dominant neighbors is approximately two thirds of the target radius. We believe that the average distance will be closer to the target radius if density is further increased from the used value 50. 


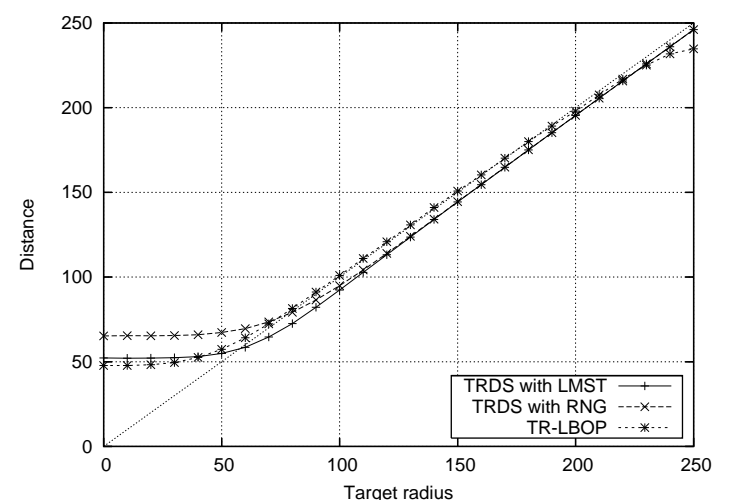

(a) Average chosen radius

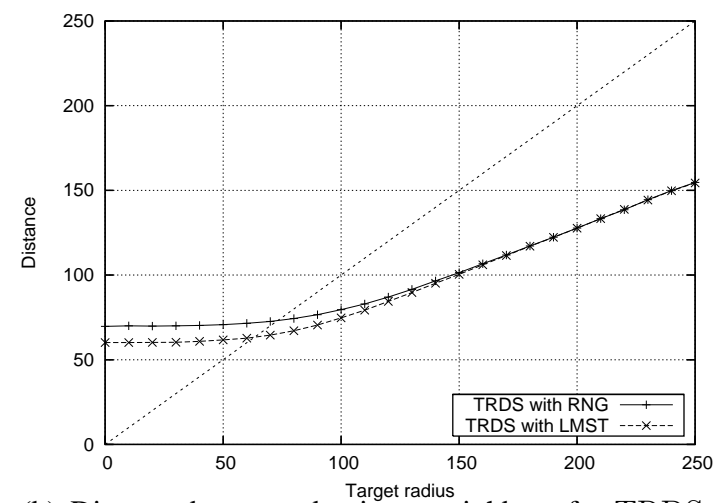

(b) Distance between dominant neighbors for TRDS

Fig. 9. Efficiency of the topology control in TR-LBOP and TRDS.

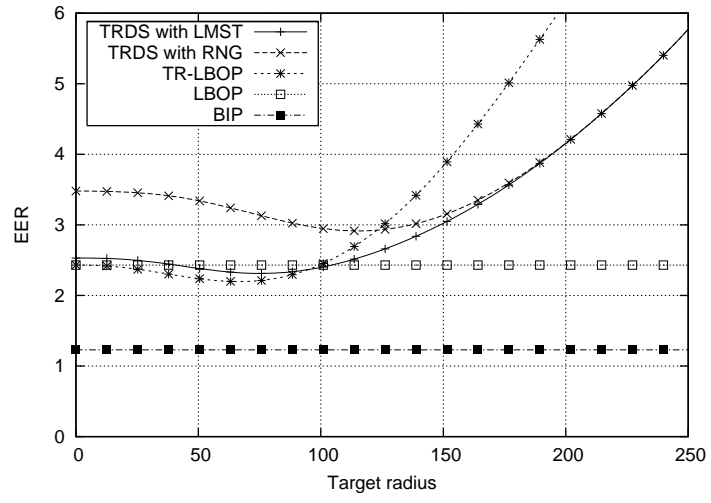

Fig. 10. EER for varying $T$.

Figure 10 illustrates the energy savings offered by our protocols, compared to other existing ones, by providing the EER obtained for varying $T$. Performances of LBOP and BIP do not vary, because their behavior is independent of the value of $T$. One can observe the existence of a minimum energy consumption in both TR-LBOP and TRDS, which is an experimental proof of the existence of an optimal radius. This minimum is obtained when $T=80 \mathrm{~m}$. (average radius of almost $80 \mathrm{~m}$.) for TR-LBOP and TRDS with LMST. This difference with the theoretical value $(100 \mathrm{~m}$.) can be explained by low density used (50) and border effects. When we increased density to 100 , the optimal radius we obtained was $96 \mathrm{~m}$. Therefore we believe that with the further increased density the practical optimal radius will approach the theoretical one. Using the LMST as a connected subgraph instead of the RNG for TRDS gives better results, and this seems natural as the LMST is itself a subgraph of the RNG: it can only be less 'demanding' than the latter.

Regarding performances, not surprisingly, centralized BIP obtains the best results, but other protocols perform well considering they depend only on local informa-

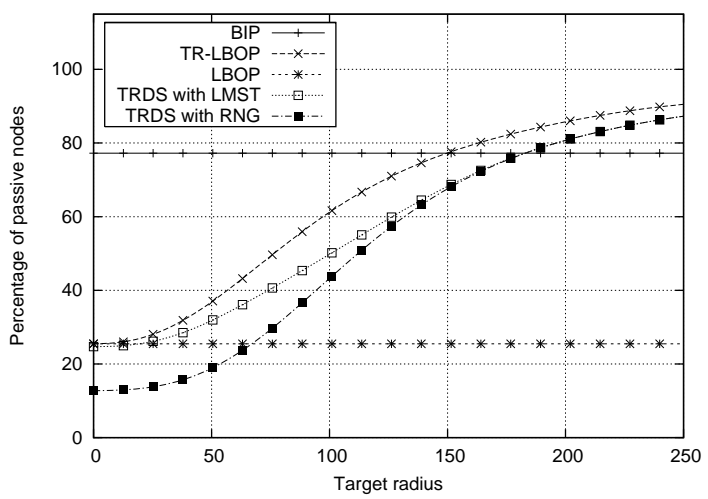

Fig. 11. Passive nodes for varying $T$.

tion about the network. The minimum consumption of TR-LBOP and TRDS with LMST is lower than the consumption of LBOP, which validates our process. The difference of energy savings between our two new protocols is very small, but if we consider that nondominant nodes can enter a sleep mode, TRDS can offer better results for some kinds of networks (like sensor networks), as these savings are not taken into account here.

Figure 11 gives the average percentage of passive nodes for both localized protocols. All non-transmitting nodes are counted, regardless of their status (dominant or not). In fact, NES (neighbor elimination) has little impact on the performance of TRDS, because the constructed network is already sparse. For instance, at density $50, T=80$, NES gives EER of 2.16 instead of 2.20. In TR-LBOP, passive nodes are idle (nodes that receive the message but do not relay it) while in TRDS they may sleep (non dominant nodes). If we consider a value of 80 for the target radius, which is the one that gives the best energy savings for both protocols, we can observe that TR-LBOP has a higher number of idle nodes than TRDS has sleeping nodes 
( $52 \%$ against $42 \%$ ). However, sleeping nodes consume far less energy than idle ones. Indeed, we assign energy values for the different states derived from [29]: sleeping nodes consume 1 unit of energy, idle nodes 15 units and transmitting nodes 28 units. As assigned radii are approximately the same for both two protocols, we can assume that a node consumes the same energy for a transmission, regardless of the protocol. For the target radius of $80 \mathrm{~m}$., and 300 nodes in the network, we obtain a consumption of 4998 units for TRDS and 6372 units for TR-LBOP. This illustrates the superiority of having sleeping nodes instead of idle ones.

This part is especially targeted at sensor networks, in which communications can be scheduled. TRDS protocol allows such a scheduling, because we know exactly which nodes have to retransmit the broadcasting messages: sleeping nodes can periodically wake up at agreed times to receive broadcast messages from their awake neighbors. This is not possible with protocols in which nodes have to monitor their neighborhood in order to achieve the diffusion. With TRDS, the 'broadcasting infrastructure' only needs to be updated from time to time to balance the load of the network (this reorganisation can also be scheduled in such networks).

Based on the computations for many densities, we give in Figure 12 the best EER obtained for each of theses densities. We can notice in 12(a) that TR-LBOP and TRDS become much more energy-efficient than LBOP with higher densities. We give also in 12(b) the overhead in the energy consumption compared to BIP. A value of 150 for LBOP and for a density of 90 means that the energy consumption of LBOP is $150 \%$ higher than the consumption of BIP for the same density. We can notice that while the overhead is constantly increasing for LBOP past a given density (around 30), the overhead of TR-LBOP is very stable and stays under $60 \%$. The overhead of TRDS is also stable past the density of 80 .

\section{CONCLUSION}

Several localized broadcasting protocols were proposed recently, with the goal of minimizing the energy consumption, while still guaranteeing a total coverage of the network. However, they were based on selecting near neighbors from a sparse topology, which did not reflect well the problems associated with the constant energy charge to each transmission. For dense networks, it resulted in energy inefficient solutions. This article settled this problem by presenting the concept of optimal transmission radius for broadcasting, computed with a hexagonal tiling of the network area. Computations were made for two different situations, with or without taking into account the energy consumption upon reception. To take advantage of this contribution, we then presented two different energy efficient protocols, named TR-LBOP and TRDS that use a target radius for topology control, instead of simply minimizing needed radii. These protocols were shown to be efficient and competitive with a centralized one like BIP, for all network densities.

The TRDS protocol is presented because it gives a unique solution to two different problems: minimum energy broadcasting and activity scheduling. By placing nodes not selected for connected dominating set into sleep mode, additional significant savings are obtained. TRDS is shown to be competitive to TR-LBOP even with all nodes remaining awake.

As part of our future work, we aim at implementing our protocols under NS2 [30]. This will allow us to obtain figures when considering mobility and a realistic MAC layer. We are also working on a localized version of BIP, in which each node apply the BIP scheme on its 2-hop neighborhood and forward the given instructions with the broadcasting packet [31]. From a certain point of view, this protocol can be seen as a variant of MPR: nodes not only forward which neighbors have been chosen as relays, but also which radius should be considered. We expect this protocol to provide really good performances, provided that 2-hop information is available at each node, which can be a drawback not present with protocols presented in this paper.

\section{ACKNOWLEDGEMENTS}

The authors greatly appreciate the efforts made by referees who carefully read the submitted version and sent us a list of remarks which contributed to substantially improved presentation. This research is partially supported by NSERC, EGIDE and INRIA.

\section{REFERENCES}

[1] D. Johnson, D. Maltz, and Y.-C. Hu, "The dynamic source routing protocol for mobile ad hoc networks (DSR)," Internet Draft, draft-ietf-manet-dsr-10.txt, July 2004, work-in-progress.

[2] A. Clementi, A. Ferreira, P. Penna, S. Perennes, and R. Silvestri, "The minimum range assignment problem on linear radio networks," in European Symposium on Algorithms (ESA 2000), Sarbrüken, Germany, September 2000.

[3] J. Wieselthier, G. Nguyen, and A. Ephremides, "On the construction of energy-efficient broadcast and multicast trees in wireless networks," in Proceedings of the IEEE Infocom 2000, Tel Aviv, Israel, March 2000.

[4] J. Cartigny, F. Ingelrest, D. Simplot-Ryl, and I. Stojmenović, "Localized LMST and RNG based minimum-energy broadcast protocols in ad hoc networks," Ad hoc Networks, vol. 3, no. 1, pp. 1 - 16, January 2004. 


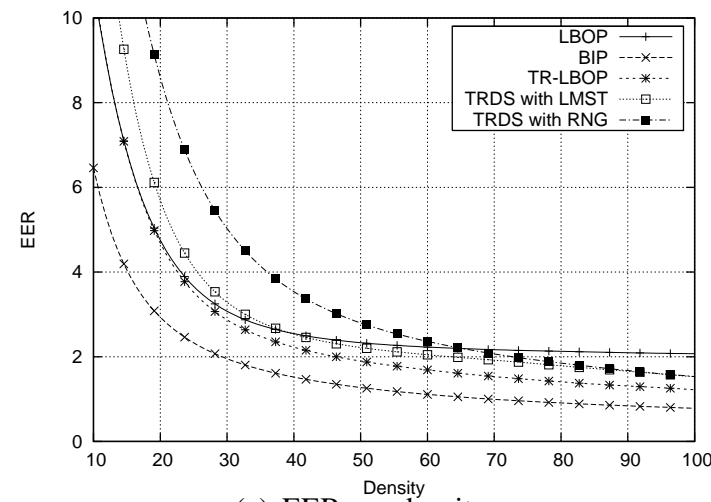

(a) EER vs. density

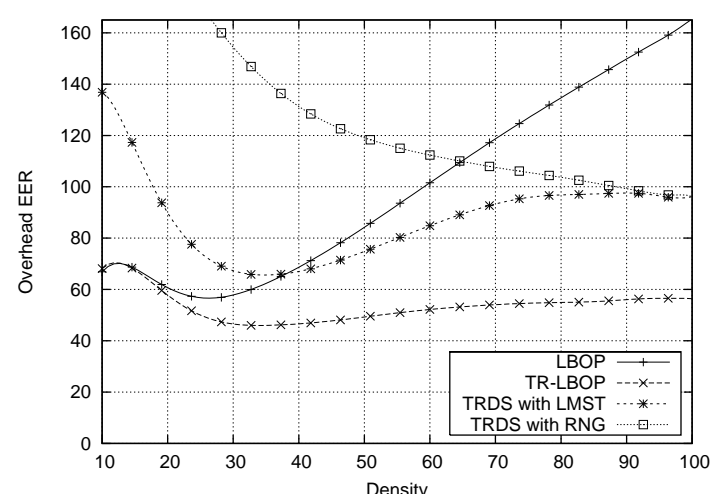

(b) Overhead EER vs. density

Fig. 12. Efficiency for varying densities.

[5] F. Ingelrest, D. Simplot, and I. Stojmenović, "Target transmission radius over LMST for energy efficient broadcast protocol in ad hoc networks," in Proceedings of the IEEE International Conference on Communications (ICC'04), Paris, France, June 2004.

[6] F. Ingelrest, D. Simplot-Ryl, and I. Stojmenović, "A dominating sets and target radius based localized activity scheduling and minimum energy broadcast protocol for ad hoc and sensor networks," in Proceedings of the Mediterranean Ad Hoc Networking Workshop (Med-Hoc-Net 2004), Bodrum, Turkey, June 2004.

[7] P. Bahl and V. Padmanabhan, "RADAR: an in-building RFbased user location and tracking system," in Proceedings of the IEEE Infocom 2000, Tel Aviv, Israel, March 2000.

[8] A. Benlarbi-Delaï, D. Simplot, J. Cartigny, and J.-C. Cousin, "Using 3D indoor microwave phase sensitive stereoscopic location system to reduce energy consumption in wireless ad hoc networks," in Proceedings of the Smart Objects Conference (sOc'2003), Grenoble, France, May 2003.

[9] D. Niculescu and B. Nath, "Ad hoc positioning system (APS) using AOA," in Proceedings of the IEEE Infocom 2003, San Francisco, USA, April 2003.

[10] N. Priyantha, A. Chakraborty, and H. Balakrishnan, "The cricket location-support system," in Proceedings of the International Conference on Mobile Computing and Networking (MobiCom 2000), Boston, USA, August 2000.

[11] V. Rodoplu and T. H. Meng, "Minimum energy mobile wireless networks," IEEE Journal on Selected Areas in Communications, vol. 17, no. 8, pp. 1333 - 1344, August 1999.

[12] J. Cartigny, D. Simplot-Ryl, and I. Stojmenović, "An adaptive localized scheme for energy efficient broadcasting in ad hoc networks with directional antennas," in Proceedings of the International Conference on Personal Wireless Communications (PWC 2004), Delft, The Netherlands, September 2004.

[13] A. Qayyum, L. Viennot, and A. Laouiti, "Multipoint relaying for flooding broadcast messages in mobile wireless networks," in Proceedings of the Hawaii International Conference on System Sciences (HICSS'02), Big Island, Hawaii, January 2002.

[14] P. Jacquet, P. Mühlethaler, T. Clausen, A. Laouiti, A. Qayyum, and L. Viennot, "Optimized link state routing protocol for ad hoc networks," in Proceedings of the IEEE International Multitopic Conference (INMIC'01), Lahore, Pakistan, December 2001.

[15] I. Stojmenović and M. Seddigh, "Broadcasting algorithms in wireless networks," in Proceedings of the International Conference on Advances in Infrastructure for Electronic Business,
Science, and Education on the Internet SSGRR, L'Aquila, Italy, July 2000.

[16] W. Peng and X. Lu, "On the reduction of broadcast redundancy in mobile ad hoc networks," in Proceedings of the ACM MobiHoc 2000, Boston, USA, August 2000.

[17] J. Cartigny, F. Ingelrest, and D. Simplot, "RNG relay subset flooding protocols in mobile ad hoc networks," International Journal of Foundations of Computer Science (IJFCS), vol. 14, no. 2, pp. 253 - 265, April 2003.

[18] G. Toussaint, "The relative neighborhood graph of finite planar set," Pattern Recognition, vol. 12, no. 4, 1980.

[19] A. Amis, R. Prakash, T. Vuong, and D. Huynh, "Max-min Dcluster formation in wireless ad hoc networks," in Proceedings of the IEEE Infocom 2001, Anchorage, Alaska, April 2001.

[20] F. Dai and J. Wu, "Distributed dominant pruning in ad hoc networks," in Proceedings of the IEEE International Conference on Communications (ICC'03), Anchorage, Alaska, May 2003.

[21] I. Stojmenović and J. Wu, "Broadcasting and activityscheduling in ad hoc networks," Ad Hoc Networking, 2004, (S. Basagni and M. Cont and S. Giordano and I. Stojmenovic, eds.) To appear.

[22] J. Carle and D. Simplot-Ryl, "Energy efficient area monitoring by sensor networks," IEEE Computer Magazine, vol. 37, no. 2, pp. $40-46,2004$.

[23] J. Shaikh, I.Stojmenović, and J. Wu, "New metrics for dominating set based energy efficient activity scheduling in ad hoc networks," in Proceedings of the International Workshop on Wireless Local Networks (WLN 2003), Bonn, Germany, October 2003.

[24] N. Li, J. C. Hou, and L. Sha, "Design and analysis of an MSTbased topology control algorithm," in Proceedings of the IEEE Infocom 2003, San Francisco, USA, April 2003.

[25] F. Ovalle-Martineza, I. Stojmenović, F. Garcia-Nocetti, and J. Solano-Gonzalez, "Finding minimum transmission radii and constructing minimal spanning trees in ad hoc and sensor networks," Journal of Parallel and Distributed Computing, vol. 65 , no. 2, pp. 132 - 141, February 2005.

[26] J. Cartigny, D. Simplot, and I. Stojmenović, "Localized minimum-energy broadcasting in ad hoc networks," in Proceedings of the IEEE Infocom 2003, San Francisco, USA, April 2003.

[27] C. Adjih, P. Jacquet, and L. Viennot, "Computing connected dominated sets with multipoint relays," INRIA, Tech. Rep. 4597, October 2002, Ad Hoc \& Sensor Wireless Networks, An Int. J., to appear.

[28] J. Wu and H. Li, "A dominating-set-based routing scheme in 
ad hoc wireless networks," in Proceedings of the International Workshop on Discrete Algorithms and Methods for Mobile Computing and Communications (DIALM), Seattle, USA, August 1999.

[29] L. Feeney and M. Nilsson, "Investigating the energy consumption of a wireless network interface in an ad hoc networking environment," in Proceedings of the IEEE Infocom 2001, Anchorage, Alaska, April 2001.

[30] NS2, http://www.isi.edu/nsnam/ns/.

[31] F. Ingelrest and D. Simplot-Ryl, "Localized broadcast incremental power protocol for wireless ad hoc networks," INRIA, Tech. Rep. 0290, January 2004. 